\title{
Abdominal Wall Endometriosis: Clinical Presentation And Imaging Features with Emphasis on Sonography
}

\author{
QUORRATA EYNUL FORHAD ${ }^{1}$, ALI AKBAR BISWAS ${ }^{2}$, SK. MONIRUL ISLAM ${ }^{3}$, MD. SHAH ALAM $^{4}$
}

\begin{abstract}
:
Objective(s): The purpose of our study was to evaluate clinical findings and sonographic features of abdominal wall endometriosis and also to report its CT and MRI appearance.

Materials and methods: A retrospective study was performed from January 2008 - December 2012, yielding 12 surgically proven cases of abdominal wall endometriosis. All patients had undergone sonography including power Doppler examination. Additional CT was performed in one case and MRI in four. Pathological material was preoperatively obtained by sonographically guided puncture in six patients. The clinical data were analyzed and the imaging studies were reviewed by radiologist working in consensus.

Results: All patients had a history of at least one prior cesarean section. All patients' C/S was done in this institution and $1500 \mathrm{C} / \mathrm{S}$ were performed within a period of 5 years giving a frequency of $0.8 \%$ scar endometriosis after C/S. All presented with focal pain near the surgical scar, which was cyclic in three patients. Nine patients presented with a palpable mass near the scar. Sonography detected 11 lesions within the abdominal wall with a mean diameter $25 \mathrm{~mm}$ and in one case sonography could not identify any lesion. All lesions were hypoechoic, vascular, and solid, with some cystic changes in one. MRI CT scan showed enhancement of the lesions. Finally histopathologically all were proved as endometriosis'.
\end{abstract}

Conclusion: Abdominal wall endometriosis frequently presents with noncyclic symptoms. Imaging findings of a solid mass near a cesarean section scar strongly suggest its diagnosis.

Keywords: abdominal wall, cesarean delivery, endometriosis, soft-tissue neoplasm

\section{Introduction:}

Endometriosis is defined as functioning endometrial tissue outside the uterine cavity. It occurs in up to $15 \%$ menstruating women ${ }^{1}$ and, in most cases, is located within the pelvis. Endometrial implants, however, have been reported in many unusual sites outside the pelvis including the abdominal wall. The latter may occur after surgical procedures that violate the uterine cavity, such as a cesarean section, allowing endometrial tissue to be transplanted ${ }^{1-5}$.

Endometriosis of the abdominal wall may be difficult to diagnose; it is often mistaken - both clinically and with diagnostic imaging - for other abnormal conditions such as a suture granuloma, an incisional hernia, or primary or metastatic cancer ${ }^{2-5}$. This may be partly due to the fact that abdominal wall endometriosis is a comparatively unknown entity that has scarcely received attention in the radiologic literature.

The goal of our study was to describe the clinical presentation and sonographic features of abdominal wall endometriosis and also to present its $\mathrm{CT}$ and MR appearance.

Materials \& Methods:We conducted a computerized study of our 500 bed hospital over a 5 year period (2008-2012) for cases of endometriosis of abdominal wall. We found 12 pathologically proven cases that were retrospectively studied.

1. Associate prof. Gynae and Obs.-Shahabuddin Medical College Hospital

2. Prof. of Surgery- Shahabuddin Medical College Hospital

3. Prof. of Radiology- Shahabuddin Medical College Hospital

4. Ex-prof. Gynae and Obs.-Dhaka Medical College Hospital 
Sonographic examination was performed in all patients. Power Doppler sonography was used to assess the vascularity of all lesions. In addition to sonography, a CT examination that included IV contrast material was performed in one patient. Four other patient also underwent MRI.

Pathologic material was preoperatively obtained by sonographically guided fine-needle aspiration (FNA) in six patients, and an additional sonographically guided histologic biopsy was performed in two of these patients. All patients underwent an operation, with surgical removal of the affected part of the abdominal wall. In all cases the diagnosis was pathologically made by the presence of endometrial stroma glands within the soft tissue and muscles of the abdominal wall, along with inflammatory cells and surroundings fibrosis $^{6}$ (Fig.1).

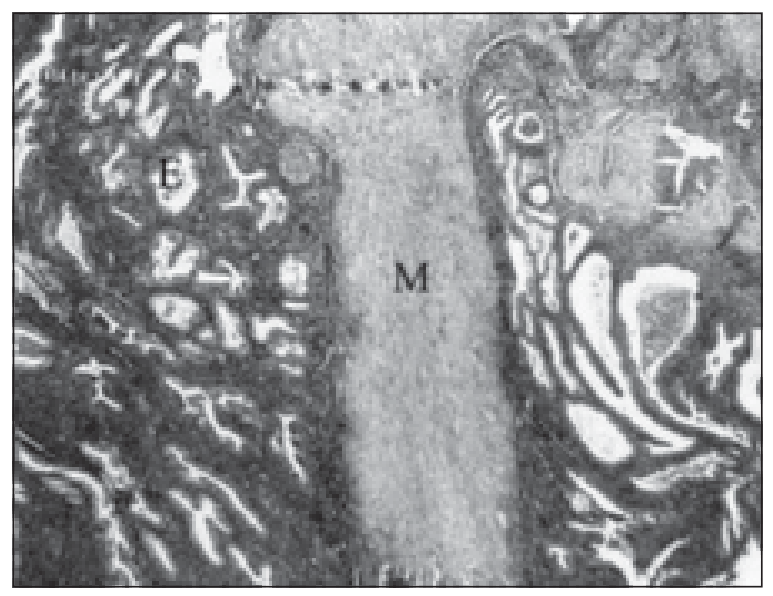

Fig.-1: Microscopic appearance of abdominal wall endometriosis in 25 years old woman. Photomicrograph shows endometriotic glands (E) next to muscular tissue of abdominal wall (M).

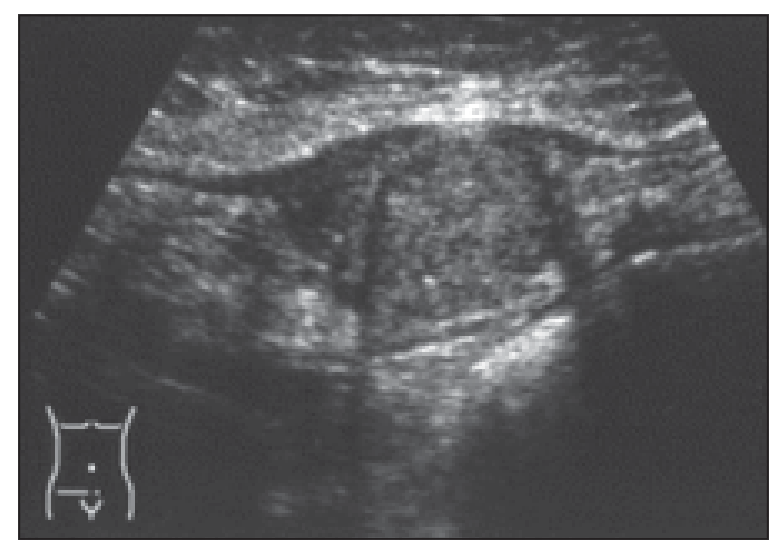

Fig.-2A: 29 years- old woman with abdominal wall endometriosis. Sonogram shows hypoechoic solid 3$\mathrm{cm}$ mass with scattered internal echoes. Mass is confined to rectus abdominis sheath.

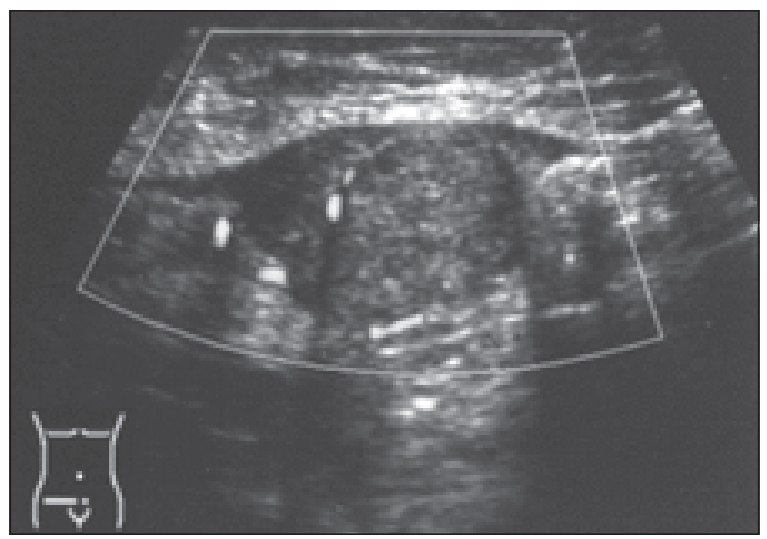

Fig.-2B: 28 years- old woman with abdominal wall endometriosis. Power Doppler sonography reveals internal vascularity within lesion.

We analyzed the clinical data of our study group, and all imaging studies were reviewed by radiologist working in consensus. Data collection included tumor site, tumor size tumor morphology, echogenicity of the tumor with respect to the predominant adjacent tissues, and detectability of intralesional blood flow with power Doppler sonography, and CT and MRI appearance and enhancement .Our institutional review broad approved this study.

\section{Results:}

The ages of the 12 women in our study group ranged from 23 to 43 years, with the mean age being 34 years. All presented with focal pain in the lower abdomen: right-sided in eight, left-sided in three, and midsagittal in one. The average time between onset of symptoms and first presentation was 12 days (range, 2 days-22 days). The pain was reported to be cyclic in three patients (25\%), increasing during menses and seldom occurring between cycles. None of the women had a history of pelvic endometriosis or reported any other complaints. All had a history of a cesarean section with Pfannenstiel's incision, and two patients had two cesarean deliveries. The average time between the cesarean section and the onset of symptoms was 4 years 3 months (range, 2 years 4 months- 6 years 4 months). In nine patients (75\%) a palpable abdominal mass was found in the region of maximum tenderness. In all cases, the location of the pain and, if present, the palpable mass were under the scar or close to the scar of the cesarean section-that is, within a maximum distance of $5 \mathrm{~cm}$ from the incision site. Characteristics of patients with age variable, interval between primary operation and development of symptoms, diagnostic aids are shown in the Table-l.

The patients were referred to the radiology department by the outpatient surgical clinic $(n=8)$, the outpatient 
Table-I

Patient's characteristics.

\begin{tabular}{lcc}
\hline Parameter & Number & Percentage (\%) \\
\hline Age (years) & 5 & 41.67 \\
23-33 & 7 & 58.34 \\
$34-43$ & & \\
Interval between primary operation and & 3 & 25 \\
development of symptoms & 2 & 16 \\
1) 2 years 4 months to 3 years 4 months & 4 & 33.34 \\
2) 3 years 5 months to 4 years 4 months & 3 & 25 \\
3) 4 years 5 months to 5 years 4 months & & \\
4) 5 years 5 months to 6 years 4 months & & 25 \\
- Cyclic & 3 & 75 \\
Pymptoms: Non-cyclic & 9 & 75 \\
Pain & 9 & 25 \\
Palpable lump & 3 & \\
No palpable lump & & 91.67 \\
Diagnostic Aid: & 11 & 91.67 \\
Sonography & 11 & 100 \\
CT and MRI & & \\
Confirm diagnosis: & 12 & \\
Histopathology & & \\
\hline
\end{tabular}

gynecologic clinic $(n=2)$, or the emergency department $(n=2)$. The presumptive clinical diagnosis before radiologic consultation was incisional hernia $(n=5)$, abdominal wall endometriosis $(n=2)$, appendicitis ( $n$ $=2)$, hematoma $(n=1)$, neurinoma $(n=1)$, or suture granuloma $(n=1)$.

Sonography showed the endometrioma in 11 patients, whereas the lesion could not be found in one case.

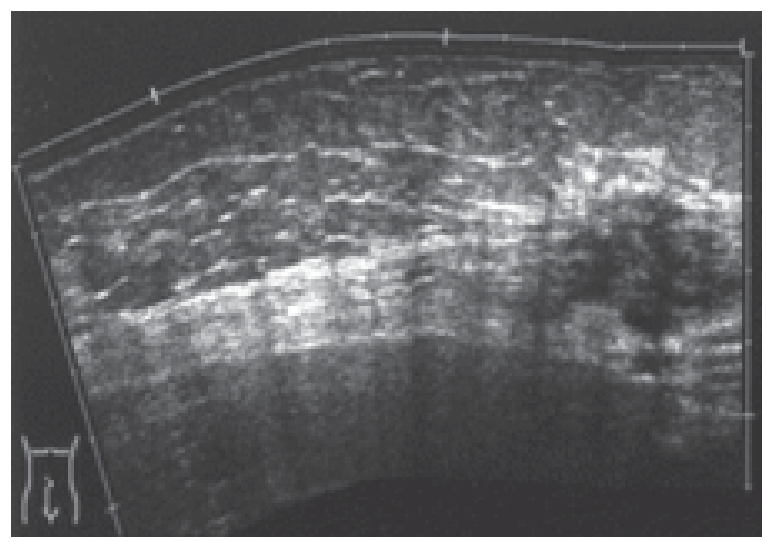

Fig.-3 A: 41-year-old woman with abdominal wall endometriosis Sonogram reveals heterogeneous hypoechoic solid mass infiltrating in both muscular abdominal wall and subcutaneous fat.
On sonography, five lesions were confined to the rectus sheath, three were located in the subcutaneous fat, and three infiltrated both of these layers. The size of the lesions ranged from 15 to $55 \mathrm{~mm}$ with a mean of $25 \mathrm{~mm}$. All masses were hypoechoic and heterogeneous with scattered internal echoes (Figs. 2A, 2B, 3A, 3B, 3C, and 3D). Ten masses were completely solid on sonography, whereas one lesion

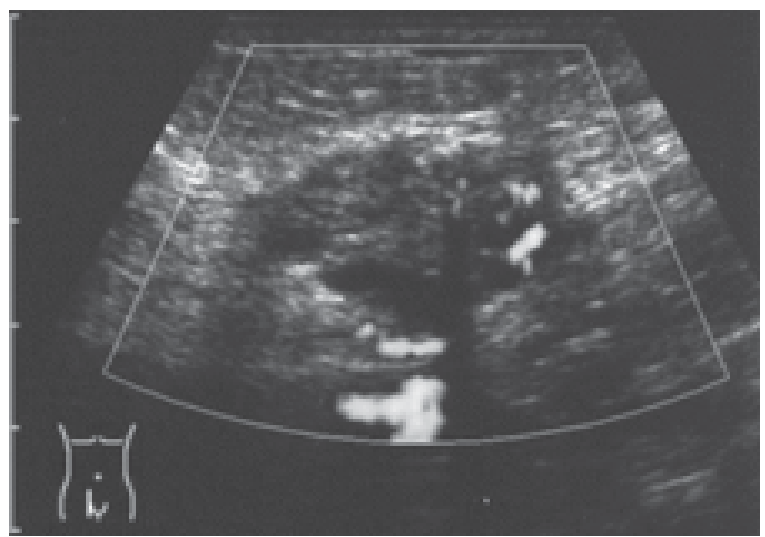

Fig.-3 B: 41- year-old woman with abdominal wall endometriosis. Power Doppler sonogram shows internal vascularity. 


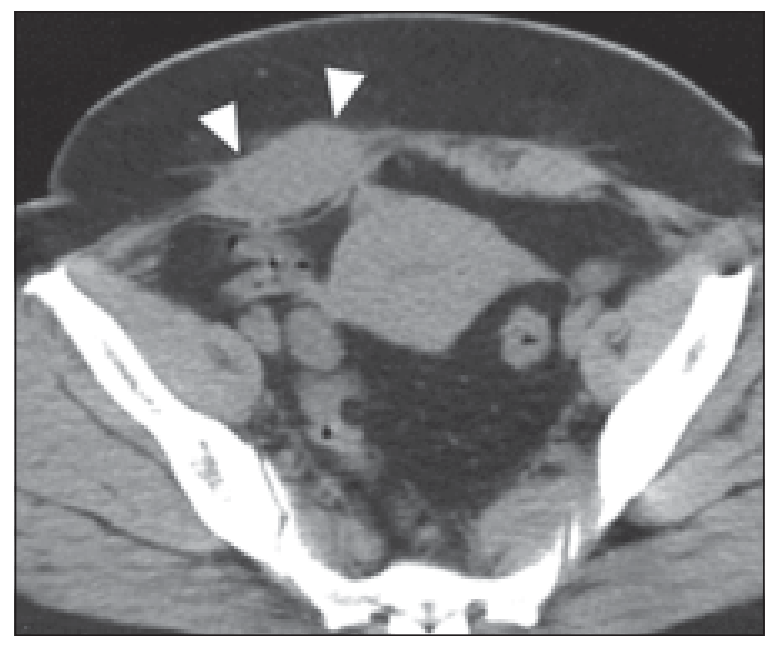

Fig.-3 C: 41- year-old woman with abdominal wall endometriosis. Unenhanced CT shows lesion (arrowheads) in right rectus sheath to be isodense to muscular tissue.

also contained some cystic changes. None of the lesions showed hyperechoic regions or calcifications. Internal vascularity was noted in all cases (Figs.2A, 2B, 3A, 3B, 3C, and 3D).

At CT examination, performed to evaluate the extent of the mass in the abdominal wall, the lesion appeared solid, ill-defined, and isodense compared with muscle and showed slight enhancement (Figs.3A, 3B, 3C, and $3 D$ ). MRI depicted the lesions as isointense to muscle on the T1-weighted sequence, as high signal

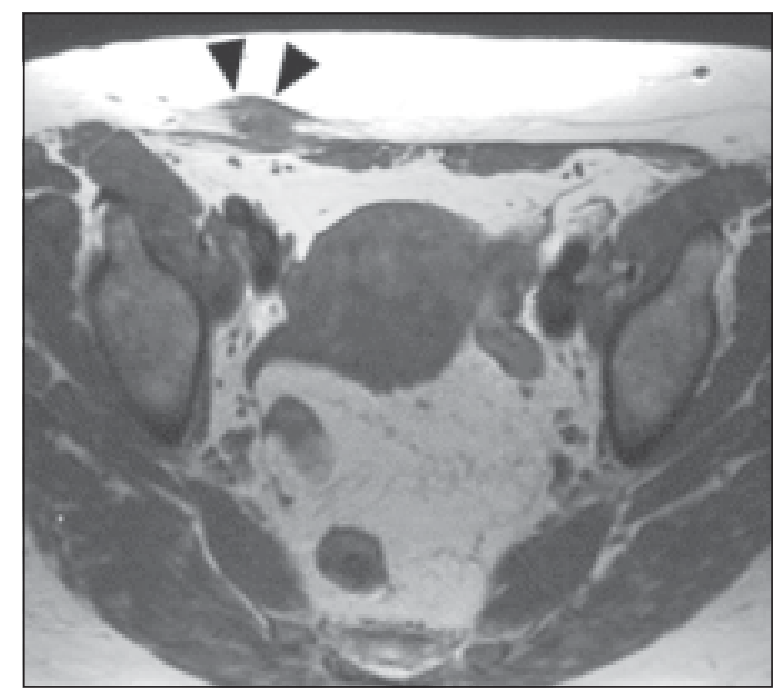

Fig.-4A: 43-year-old woman with abdominal endometriosis. Axial T1-weighted spin-echo MR image shows lesion (arrowheads) in subcutaneous fat of right lower abdominal wall. Lesion is isointense to muscle.

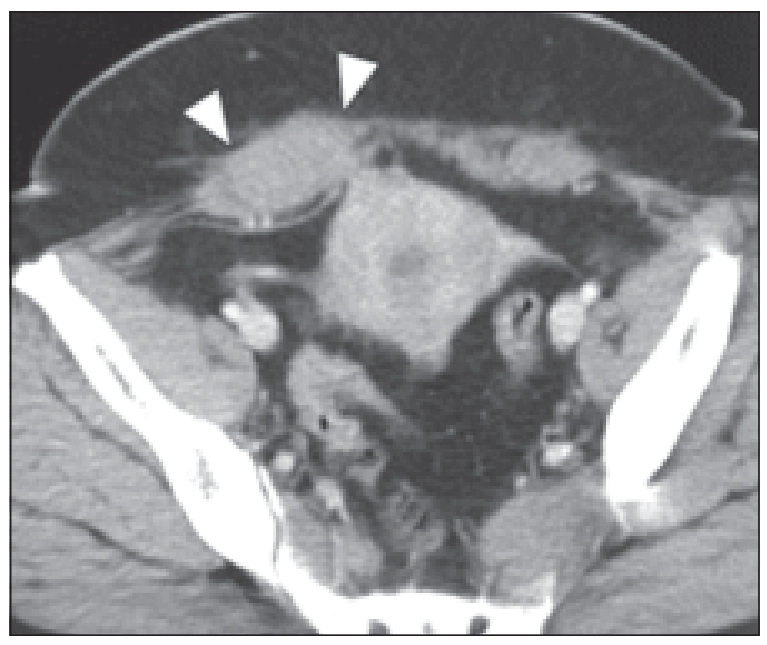

Fig.-3 D: 41-year-old woman with abdominal wall endometriosis. Contrast- enhanced CT shows slight enhancement of lesion (arrowheads).

intensity on fat-saturated T2-weighted images, and with marked enhancement (Figs.4A, 4B, and 4C).

Two patients were initially treated with hormone therapy, receiving progestogens orally, without sufficient results. Finally all patients underwent surgery with complete excision of the masses. At surgery, the size of the endometriomas was similar to the sonographic measurements, and the sonographically missed lesion had a diameter $(20 \mathrm{~mm})$ within the range of the other masses. One patient had a recurrence of

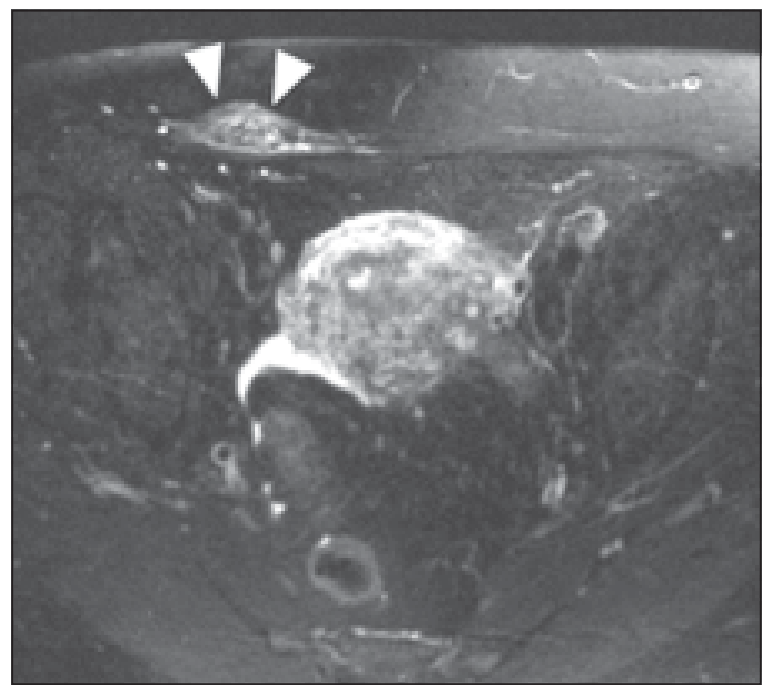

Fig.-4B: 43-year-old woman with abdominal wall endometriosis. Axial T2- weighted fat-saturated fast spin-echo MR image depicts lesion (arrowheads) as heterogeneous high-signal-intensity mass. 


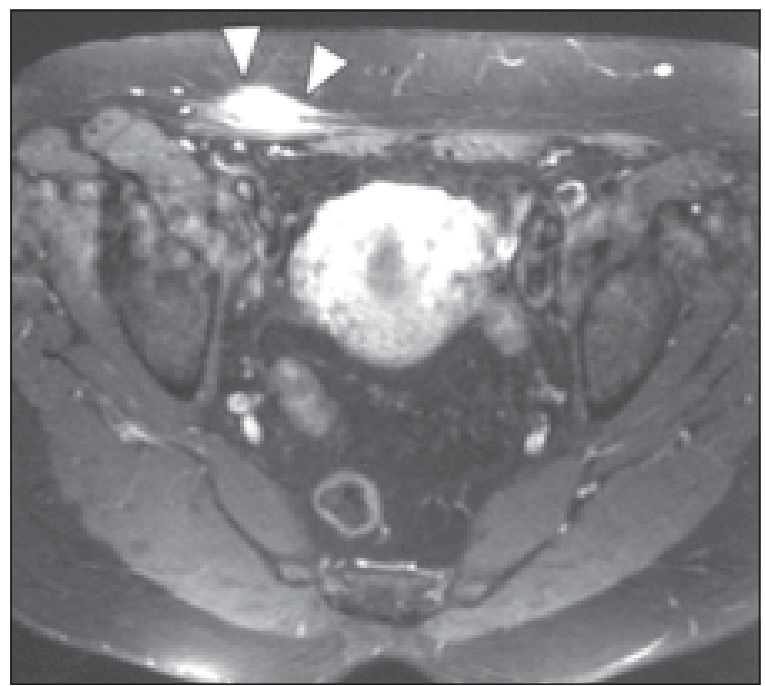

Fig.-4C: 43-year- old woman with abdominal wall endometriosis. Axial T1- weighted fat-saturated spinecho MR image after injection of contrast medium shows strong enhancement of lesion (arrowheads).

abdominal wall endometriosis 30 months after resection.

In the 5-year study period, approximately 1,500 cesarean sections were performed in our hospital. Given our study group of 12 women, the calculated frequency of abdominal wall endometriosis is approximately $0.8 \%$ of all women who had a cesarean delivery.

\section{Discussion:}

Endometriosis is a relatively common gynecologic problem in women of reproductive age. Two leading theories exist for its cause; one hypothesis suggests that mesenchymal cells with retained multipotential may, under the proper circumstances, undergo metaplasia into endometriosis. The other theory states that endometrial cells may be transported to ectopic sites forming an endometrioma ${ }^{1-4}$. When stimulated by estrogens, these cells may proliferate until they become symptomatic.

Extrapelvic endometriosis has been described in nearly all body cavities and organs, but its most frequent location is in the abdominal wall ${ }^{7}$. Although an incidental case of spontaneous endometriosis in a scarless abdominal wall has been reported ${ }^{7}$, it is usually associated with operations in which the uterus is opened ${ }^{1-6}$. In all our patients the endometriomas developed in association with a cesarean section sear and in the absence of any history of pelvic endometriosis, thereby supporting the etiologic concept of iatrogenic transport. We found abdominal wall endometriosis to be present in approximately 0.8 $\%$ of all women who had cesarean deliveries at our institution, which appears more frequent than is generally assumed but lies within the range of rates described in the published literature (0.03-1 \%) $)^{4,8}$. The true incidence rate is probably higher than $0.8 \%$, as some cases of abdominal wall endometriosis may have remained undetected because our study did not include routine follow-up in all women after a cesarean section.

In general, the characteristic clinical symptom of endometriosis is cyclic pain associated with menses 8,9 . All our patients with abdominal wall endometriosis presented with focal abdominal pain; however, this pain was constantly present and not associated with the menstrual cycle in the majority of cases (75\%). The noncyclic nature of pain in endometriosis of the abdominal wall has occasionally been reported by others 2,5 but has generally been regarded as atypical, which may explain why it is clinically often misdiagnosed, as was the case in our patients.

Most patients also presented with a palpable mass at the site of maximum tenderness in the region of the surgical scar. Sonography showed these masses to be solid, hypoechoic lesions in the abdominal wall and to contain internal vascularity on power Doppler examination. Only one of the endometriomas also contained cystic areas. In view of the wide morphologic spectrum of endometriosis - varying from purely cystic chocolate cyst to solid deposits or fibrosis ${ }^{9}$ the almost invariably pure solid aspect of abdominal wall endometriosis in our study is remarkable. The imaging appearance might be expected to be more heterogeneous, with frequent cystic changes due to intralesional bleeding associated with menstruation. The solid aspect of the abdominal wall endometriomas in our study, however, concurs with some other imaging reports ${ }^{10,11}$.

Sonographic examination missed the abdominal wall endometrioma in one of our patients, possibly because the examiner did not focus on the near field.

These sonographic findings are nonspecific, and a wide spectrum of disorders presenting as a mass in the abdominal wall should be considered in the imaging differential diagnosis. This should include neoplasms, such as a sarcoma, desmoid tumor, lymphoma, or metastasis, and nonneoplastic causes, such as a 
suture granuloma, ventral hernia, hematoma, or abscess $[9,10]$. Sonography may be able to exclude the latter three in view of the solid appearance and vascular nature of abdominal wall endometriomas.

The CT and MR characteristics of abdominal wall endometriosis are nonspecific also, both showing a solid enhancing mass in the abdominal wall ${ }^{12,13}$. The major role of CT and MRI may be to depict the extent of the disease preoperatively.

In half of our patients, the final diagnosis was preoperatively made by sonographically guided puncture. Sonographically guided FNA is a rapid and accurate diagnostic procedure in women with abdominal wall masses associated with endometriosis, enabling malignancy to be excluded and a definitive treatment to be defined ${ }^{6}$. If the FNA results are inconclusive, as may occur because endometriomas are often fibrous in nature ${ }^{4}$, an additional histologic biopsy may be considered.

Therapeutic options for abdominal wall endometriosis are pharmacologic therapy with hormonal agents, such as progestogens, or surgical excision. The success rate of medical therapy has been reported to be low, offering only temporary alleviation of symptoms often followed by recurrence after cassation of the drug $^{2}$. Wide surgical excision therefore is the treatment of choice ${ }^{1-3}$.

Our study is limited by its retrospective nature, concerning only patients with a final diagnosis of abdominal wall endometriosis. No defined standardized protocol was used to scan our patients, which might have led to lack of uniformity. However, all patients were imaged on sonography in both the transverse and longitudinal views, and power Doppler examination was performed in all cases. We therefore believe that this limitation is minor and would not change the overall impression of our analysis.

In conclusion, abdominal wall endometriosis after a cesarean section is more frequent than generally assumed. Clinically it is often misdiagnosed because endometriosis may occur years after the cesarean section, the pain is often noncyclic in nature, and there is not always a palpable mass. The sonographic finding of a solid mass in the abdominal wall is not pathognomonic for endometriosis, but if located close to a cesarean section scar it should be of prime consideration in the differential diagnosis.

\section{References:}

1. Patterson GK, Winburn GB. Abdominal wall endometriomas: report of eight cases. Am Surg 1999; 65:36-39.

2. Koger KE, Shatney $\mathrm{CH}$, Hodge $\mathrm{K}$, McClenathan $\mathrm{JH}$. Surgical scar endometrioma. Surg Gynecol Obstet 1993; 177:243-246.

3. Seydel AS, Sickel JZ, Warner ED, Sax HC. Extrapelvic endometriosis: diagnosis and treatment. Am J Surg 1996; 171:239-241.

4. Dwivedi AJ, Agrawal SN, Silva YJ. Abdominal wall endometriomas. Dig Dis Sci 2002; 47: 456-461.

5. Blanco RG, Parithivel VS, Shah AK, Gumbs MA, Schein M, Gerst PH. Abdominal wall endometriomas. Am J Surg 2003; 185: 596-598.

6. Simsir A, Thorner K, Waisman J, Cangiarella J. Endometriosis in abdominal scars: a report of three cases diagnosed by FNA biopsy. Am Surg 2001; 67:984-986 [Medline]

7. Ideyi SC, Schein M, Niazi M, Gerst PH. Spontaneous endometriosis of the abdominal wall. Dig Surg 2003; 20:246-248.

8. Singh KK, Lessells AM, Adam DJ, et al. Presentation of endometriosis top general surgeons: a 10-year experience. Br J Surg 1995; 82:1349-1351.

9. Woodward PJ, Sohaey R, Mezzetti TP. Endometriosis: radiologic-pathologic correlation. Radio-Graphics 2001; 21: 193-216

10. Wolf $C$, Obrist $P$, Ensinger $C$. Sonographic features of abdominal wall endometriosis. AJR 1997; 169:916-917.

11. Francica G, Giardiello C, Angelone G, Cristiano S, Finelli R, Tramontano G. Abdominal wall endometriomas near cesarean delivery scars: sonographic and color Doppler findings in a series of 12 patients. J Ultrasound Med 2003; 22: 1041-1047.

12. Coley BD, Casola G. Incisional endometrioma involving the rectus abdominis muscle and subcutaneous tissues: CT appearance. AJR 1993; 160:549-550.

13. Wolf GC, Kopecky KK. MR imaging of endometriosis arising in cesarean section scar. J Comput Assist Tomogr 1989; 13: 150- 152. 